\title{
'ZipperCream-GC', a Large-seeded, Cream-type Southernpea with a Green Cotyledon Phenotype
}

\author{
Richard L. Fery ${ }^{1}$ \\ U.S. Department of Agriculture, Agricultural Research Service, U.S. \\ Vegetable Laboratory, 2700 Savannah Highway, Charleston, SC 29414- \\ 5334
}

Additional index words. Vigna unguiculata, cowpea, vegetable breeding

\begin{abstract}
'ZipperCream-GC' is a new southernpea [Vigna unguiculata (L.) Walp.] cultivar released in 2008 by the Agricultural Research Service of the U.S. Department of Agriculture. 'ZipperCream-GC' was developed as a replacement for 'Zipper Cream', a largeseeded, cream-type crowder cultivar long popular with home gardeners and fresh market growers throughout the southeastern United States. The major attributes of the new cultivar are its high yield, large seed size, and green cotyledon phenotype. 'ZipperCream-GC' can be harvested at the dry stage of maturity without loss of the pea's fresh green color.
\end{abstract}

\section{Origin}

'ZipperCream-GC' is the product of a backcross/pedigree-type breeding program initiated in 1996 (Fig. 1). The parental lines are USVL 01-951-2 and USVL 01-592-2. USVL 01-951-2 is an F3 selection from a BC2 population developed using the cultivars Zipper Cream (the recurrent parent) and Bettergreen (Fery et al., 1993; Lorz, 1970). USVL $01-592-2$ is an F3 selection from a $\mathrm{BC} 2$ population developed using the cultivars Zipper Cream (the recurrent parent) and Freezegreen (Chambliss, 1979). 'Bettergreen', a cream-type southernpea, is homozygous for the $g c$ gene conditioning the green cotyledon trait (Fery and Dukes, 1994). After the cross between USVL 01-951-2 and USVL 01-5922 , intense selection pressure was applied in the F2 though the F6 generations for the green cotyledon trait and the seed and plant habit characteristics exhibited by 'Zipper Cream'. 'ZipperCream-GC' originated as a bulk of an F6 population grown in 2003.

\section{Description}

'ZipperCream-GC' plants are similar in appearance to those of 'Zipper Cream'. 'ZipperCream-GC' matures to the dry-pod

Received for publication 14 Aug. 2008. Accepted for publication 16 Apr. 2009.

The technical assistance of Floyd P. Maguire is gratefully acknowledged.

${ }^{1}$ To whom reprint requests should be addressed; e-mail Richard.Fery@ars.usda.gov. harvest stage at Charleston, $\mathrm{SC}$, in $\approx 70$ to $74 \mathrm{~d}$ (similar to 'Zipper Cream') (Table 1). The plant habit is high bushy. The leaf color is dark green; the leaf surface is blistered and glossy. There is no obvious purple pigmentation on the stems, branches, petioles, or peduncles. Flower color is predominantly white; the bottom of the standard is yellow (the back of the standard is a light yellow color; the front exhibits a tinge of yellow color on a white background). Pod set is concentrated, and the pods are borne at the foliage level in a scattered fashion. Dry pods are attached to the peduncles in a pendant manner, and each peduncle typically produces one or two pods. A typical dry pod is moderately curved, 15 to $16 \mathrm{~cm}$ long, exhibits deep constrictions between peas, and contains 14 to 15 peas. Pod color is dark green when immature, light green (as opposed to the cream color of 'Zipper Cream' pods) when ready for mature green harvest, and light straw color when completely dry. There is a tendency for some mature green pods to exhibit a slight amount of purple pigmentation or purple blushing between peas. Like 'Zipper Cream', 'ZipperCream-GC' is highly resistant to the southern root-knot nematode [Meloidogyne incognita (Chitwood) Kofoid and White]. Fresh 'ZipperCream-GC' peas exhibit a semicrowder to crowder shape, similar to 'Zipper Cream' peas. The dry peas have a globose-like shape and a smooth

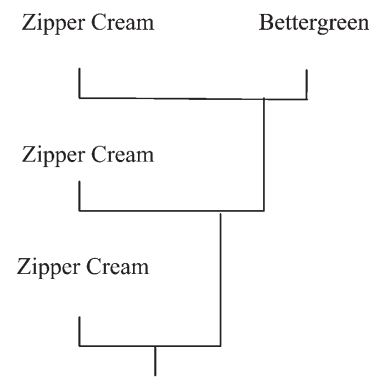

USVL 01-951-2 seedcoat and are slightly smaller than those of 'Zipper Cream' (19.6 g versus 20.3 g per 100 peas, respectively).

The results of five replicated field tests conducted over a 4-year period (2004 through 2007) at Charleston, SC, indicate that 'ZipperCream-GC' and 'Zipper Cream' yields are comparable (Table 1). No yield differences could be demonstrated between the two cultivars in three of the tests, but 'Zipper Cream' produced a higher yield in two of the tests. The mean dry seed yield over the five tests was not significantly different between the two cultivars; however, the cultivar $\times$ season interaction was significant $(P \leq 0.05)$.

'ZipperCream-GC' is homozygous for the $g c$ gene that conditions the green cotyledon trait. Seedcoats and cotyledons of fieldgrown 'ZipperCream-GC' peas harvested at the dry stage of maturity are light olive in color, whereas those of 'Zipper Cream' peas harvested at a similar stage of maturity are a cream color. 'ZipperCream-GC' peas imbibed to restore fresh-harvest seed size and blanched in boiling water for $3 \mathrm{~min}$ exhibit a near-fresh green color (Fig. 2).

'ZipperCream-GC' is recommended for use by fresh-market growers, home gardeners, and the frozen food industry. The mature green pods are extremely easy to shell. The ease-of-shelling trait is important to consumers because most home gardeners and purchasers of fresh, mature green pods must shell the pods by hand. The frozen food industry can imbibe the dry peas in water and use the resulting raw product to produce a pack that closely approximates the color and appearance of a pack prepared using fresh peas. The shape and size of 'ZipperCreamGC' seeds are quite similar to those of 'Zipper Cream' seeds; seeds of the two cultivars can be readily blended (packed together) to produce a retail product with the desired intensity of green color.

\section{Availability}

Breeder's seed of 'ZipperCream-GC' has been released to seed producers. Small samples

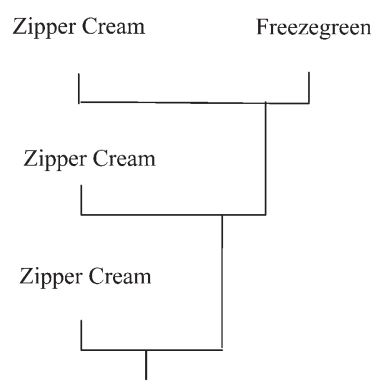

USVL 01-592-2 
Table 1. Number of days to harvest, weight per 100 dry peas, and dry pea yield for 'Zipper Cream' and 'ZipperCream-GC' cream-type southernpeas grown in five separate trials at Charleston, SC, 2004 to 2007. ${ }^{\mathrm{C}}$

\begin{tabular}{|c|c|c|c|}
\hline Trial/cultivar & Days to harvest (no.) & Wt./100 dry peas $(\mathrm{g})$ & Dry pea yield $\left(\mathrm{kg} \cdot \mathrm{ha}^{-1}\right)$ \\
\hline \multicolumn{4}{|l|}{ Trial I } \\
\hline Zipper Cream & $70.0 \mathrm{a}^{\mathrm{y}}$ & $20.7 \mathrm{a}$ & $1,758 \mathrm{a}$ \\
\hline ZipperCream-GC & $73.2 \mathrm{a}$ & $19.7 \mathrm{a}$ & $1,749 \mathrm{a}$ \\
\hline \multicolumn{4}{|l|}{ Trial II } \\
\hline Zipper Cream & $69.8 \mathrm{a}$ & $21.4 \mathrm{a}$ & $851 \mathrm{a}$ \\
\hline ZipperCream-GC & $69.8 \mathrm{a}$ & $21.1 \mathrm{a}$ & $874 \mathrm{a}$ \\
\hline \multicolumn{4}{|l|}{ Trial III } \\
\hline Zipper Cream & $72.3 \mathrm{a}$ & $22.1 \mathrm{a}$ & $770 \mathrm{a}$ \\
\hline ZipperCream-GC & $72.2 \mathrm{a}$ & $21.5 \mathrm{a}$ & $902 \mathrm{a}$ \\
\hline \multicolumn{4}{|l|}{ Trial IV } \\
\hline Zipper Cream & $72.0 \mathrm{a}$ & $20.0 \mathrm{a}$ & $2,008 \mathrm{a}$ \\
\hline ZipperCream-GC & $72.1 \mathrm{a}$ & $18.7 \mathrm{a}$ & $1,745 \mathrm{~b}$ \\
\hline \multicolumn{4}{|l|}{ Trial V } \\
\hline Zipper Cream & $74.0 \mathrm{a}$ & $17.4 \mathrm{a}$ & $2,257 \mathrm{a}$ \\
\hline ZipperCream-GC & $74.2 \mathrm{a}$ & $16.5 \mathrm{~b}$ & $1,917 \mathrm{~b}$ \\
\hline \multicolumn{4}{|c|}{ Combined analysis of all trials } \\
\hline Zipper Cream & $71.8 \mathrm{~b}^{*}$ & 20.3 a NS & $1,503 a^{*}$ \\
\hline ZipperCream-GC & $72.2 \mathrm{a}$ & $19.6 \mathrm{~b}$ & $1,410 \mathrm{a}$ \\
\hline
\end{tabular}

${ }^{2}$ Trials I through V planted on 21 May 2004, 26 May 2005, 14 June 2005, 31 May 2006, and 31 May, 2007, respectively. Experimental design of each trial was a randomized complete block with six replications (Trial I) or 10 replications (Trials II, III, IV, and V). Each plot was space-planted, 18 hills per plot, three seeds per hill, $30 \mathrm{~cm}$ between hills, and $102 \mathrm{~cm}$ between rows; the center 16 hills of each plot were hand harvested to simulate a single, once-over, mechanical harvest.

${ }^{\mathrm{y}}$ Mean separation within columns and trials by the Student-Newman-Keuls multiple range test $(P \leq 0.05)$. NS, ${ }^{*}$ Nonsignificant or significant interaction between cultivar and trial at $P \leq 0.05$, respectively.

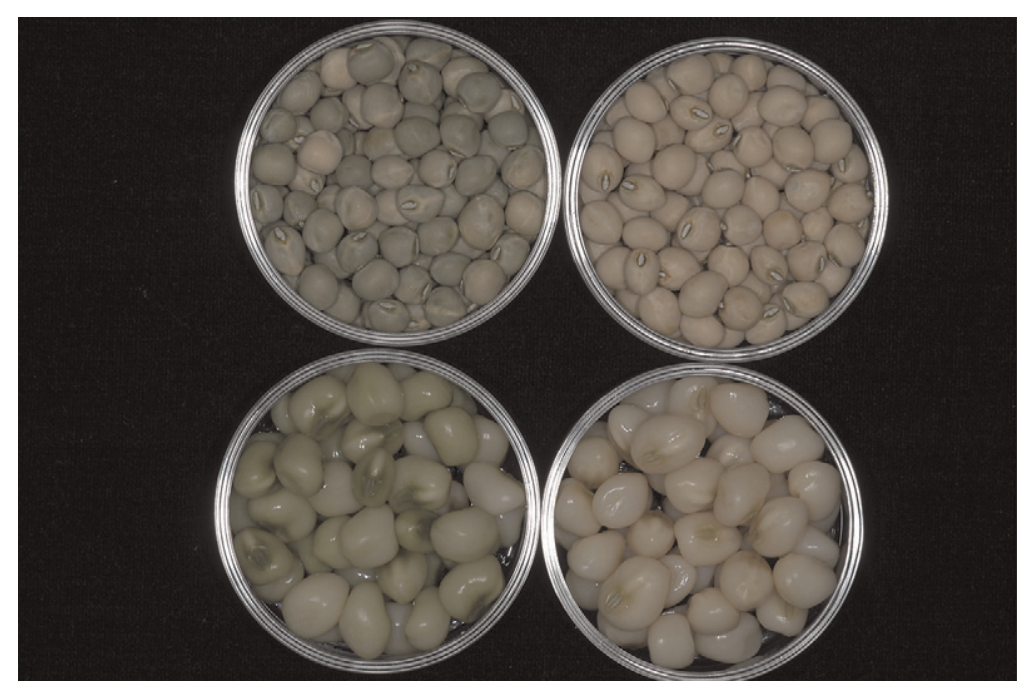

Fig. 2. Dried seeds (top) and dried seeds imbibed to restore fresh-harvest seed size and blanched in boiling water for $3 \mathrm{~min}$ (bottom) of 'ZipperCream-GC' (left) and 'Zipper Cream' (right). Note the green color of both the dried and imbibed seeds of 'ZipperCream-GC' $(g c / g c)$ and the cream color of both the dried and imbibed seeds of 'Zipper Cream' $(G c / G c)$. of 'ZipperCream-GC' breeder's seed are available from the author for distribution to interested research personnel. Genetic material of this release will be deposited in the National Plant Germplasm System where it will be available for research purposes, including development and commercialization of new cultivars. It is requested that appropriate recognition of the source be given when this germplasm contributes to research or development of a new breeding line or cultivar.

\section{Literature Cited}

Chambliss, O.L. 1979. 'Freezegreen' southernpea HortScience 14:193.

Fery, R.L. and P.D. Dukes. 1994. Genetic analysis of the green cotyledon trait in southernpea [Vigna unguiculata (L.) Walp.]. J. Amer. Soc. Hort. Sci. 119:1054-1056.

Fery, R.L., P.D. Dukes, and F.P. Maguire. 1993. 'Bettergreen' southernpea. HortScience 28: 856.

Lorz, A.P. 1970. Zipper Cream, a high producing fresh market southern pea with processing potential. Univ. Fla. Agr. Expt. Sta. Circ. S-210. 